\title{
Early-Stage Apple Leaf Disease Prediction Using Deep Learning
}

\author{
Amit Gawade 1 , Subodh Deolekar ${ }^{1,2}$ and Vaishali Patil ${ }^{2}$ \\ ${ }^{1}$ REDX Innovation Lab, Prin. L. N. Welingkar Institute of \\ Management Development and Research, Mumbai, India \\ ${ }^{2}$ Prin. L. N. Welingkar Institute of Management Development \\ and Research, Mumbai, India
}

\section{ABSTRACT}

Premature leaf fall, scab, Alternaria leaf spot, brown spot, mosaic, grey spot, and rust are common types of apple leaf diseases. Due to the arrival of monsoon, there is excess moisture in the air because of an outbreak of diseases in plants that is being witnessed in the hilly region. Farmers from these regions are always worried about the health of Apple plants. The scientists working in various departments, KrishiVigyanKendra's, and regional research stations have given the required inputs to control the problems but that is not useful to identify the problem in the early stage. Also, the current disease diagnosis based on human scouting is time-consuming and expensive. Our proposed system identifies various apple leaf diseases in an early stage that will alert the farmers and nearby research institutes to take appropriate action to control it. The dataset contains 1821 images of apple leaves which has normal leaves, scab, rust, and other disease infected leaves. The proposed regional convolutional neural network-based approach is capable of localizing and classifying the disease with $90 \%$ accuracy.

KEY WORDS: MASK RCNN, OBJECT DETECTION, LEAF DISEASE DETECTION, DEEP LEARNING, CNN.

\section{INTRODUCTION}

Agriculture plays an important role in the economy of every country. Produce produced by farmers must be in a proper condition to achieve expected profit. Advancements in technology must be used in this domain to save and make products market ready. But due to continuous change in weather and lack of technology access in this field, farmers are facing a huge challenge

Biosc Biotech Res Comm P-ISSN: 0974-6455 E-ISSN: 2321-4007

\section{crossef}

Identifiers and Pagination

Year: 2021 Vol: 14 No (5) Special Issue

Pages: $40-43$

This is an open access article under Creative

Commons License Attribn 4.0 Intl (CC-BY). DOI: $h t t p: / / d x$.doi.org/10.21786/bbrc/14.5/8 to protect their produce from different diseases that are unexpected and occurred at any time.

Traditional way of disease detection is based on observation and time consuming which requires experts to be present on the field. Sometimes misdiagnosis of many diseases may cause harm to crops, products and consumers who are consuming the product. Artificial Intelligence (AI) plays a significant role in every vertical like agriculture. AI can be useful to solve most common issues in agriculture. It can be used to identify various leaf diseases in an early stage. Using automatic plant leaf disease detection methods farmers will get help to reduce their losses and to improve the productivity. Various researchers have been working on this problem. Most of them tried machine learning, image processing techniques and various deep learning algorithms to diagnose the plant diseases. We have used Deep Learning technique which is a subset of AI to detect the apple leaf disease in an early stage.The proposed methodology generates

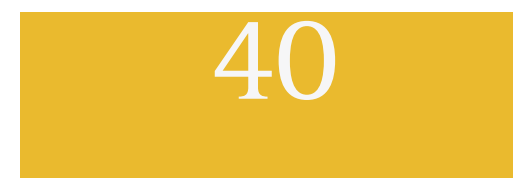


masks around the affected area of the leaf and detects disease in an efficient way. We have used a confusion matrix as a performance measure for the CNN model and bounding boxes have been drawn closest to the affected region of the plant that shows the proposed model performs well.

Literature Review: Agriculture is the primary occupation not only in India but many countries depend on it. Researchers throughout the globe are trying to solve different problems faced by farmers with the help of the latest technology. Zhong and Zhao have proposed three methods, namely multi-label classification, focus loss function and regression. These are based on DenseNet121 architecture. Total 2462 images of apple leaf were used that contained six apple leaf diseases. The proposed method achieved 93.5\% accuracy which is better than traditional multi classification techniques.

To check without using CNN can we extract features using Shallow CNN with Kernel SVM and Shallow CNN with Random Forest, Li et al. had compared these two methods with other pre-trained deep learning models on three different datasets maize, grape and apple. They had found that the above mentioned two algorithms performed well in terms of precision, recall and F1 score. Bin Tahirused a plant village dataset to re-train the Inception V3 model using transfer learning and extracted features required for classification. These features were down sampled using a novel variance-controlled approach that finds how each pixel varies from other nearest pixels. It reduces redundancy from the features. The proposed method achieved 97\% of accuracy.

Many researchers are working on detection of leaf diseases by using different datasets based on deep learning techniques. Hu et al.proposed deep CNN with multiscale feature extraction methods for tea leaf disease detection. Experimental results show that the proposed method gave 92.5\% accurate results. Iteration time required for the proposed model is less in comparison to VGG16 and AlexNet models. Detection of leaf disease, manually is a very big challenge for farmers those who are new to farming. Subetha et al. compared the performance of two algorithms ResNet50 and VGG19. Dataset was publicly available on Kaggle that contains 3651 real time images having four classes namely scab,healthy, multiple diseases and rust. As per results both architectures gave 87\% accuracy. Singh and Misraproposed an algorithm to segment the image that can be used further for classification of multiple leaf diseases. They had used a genetic algorithm to detect the leaf disease in an early stage.

\section{PROPOSED METHODOLOGY}

The proposed method is based on Mask-RCNN (Regional Convolutional Neural Network). Mask RCNN is an extension of faster RCNN. RCNN is an object detection algorithm which is also used for image segmentation and masking. It is a pixel level classification that determines which all pixels belong to which object.The Mask-RCNN architecture is divided into three parts. First pretrained model of CNN, second Region Proposal Network(RPN) and last is fully connected layer and output. Backbone to this algorithm is ResNet50 which is used to extract the features of images. Detailed pipeline of proposed method is shown in figure 1, below.

Figure 1: Proposed method based on Mask RCNN Architecture

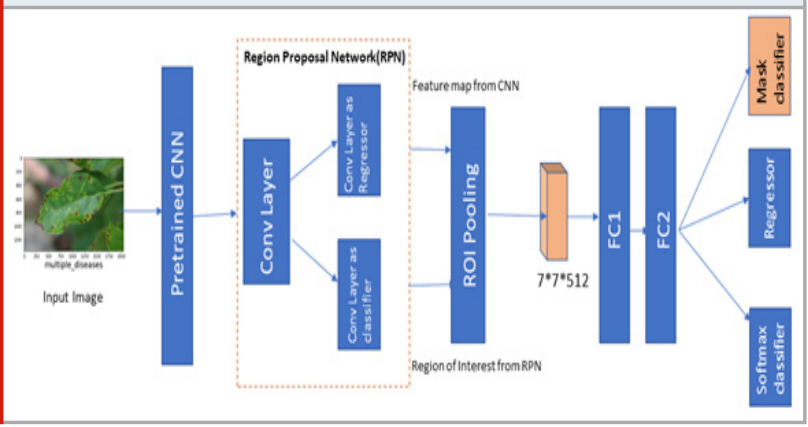

Figure 2: Samplesfrom Plant Pathology 2020 dataset.

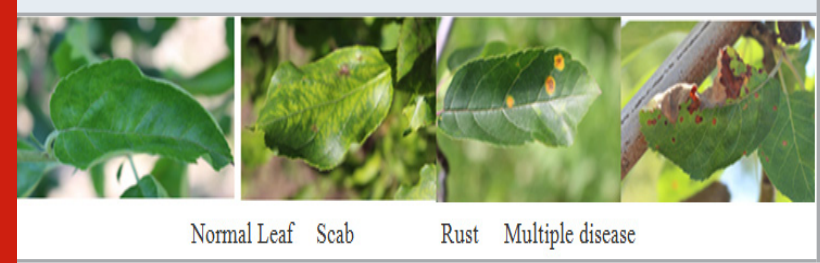

Initially we have used weights of pre trained model trained on plant pathology 2020 dataset. Last layer of this pretrained model architecture is a fully connected layer that has a softmax activation function. Feature maps from this model is used as an input to the Region Proposal network.

Figure 3: VGG image Annotator tool

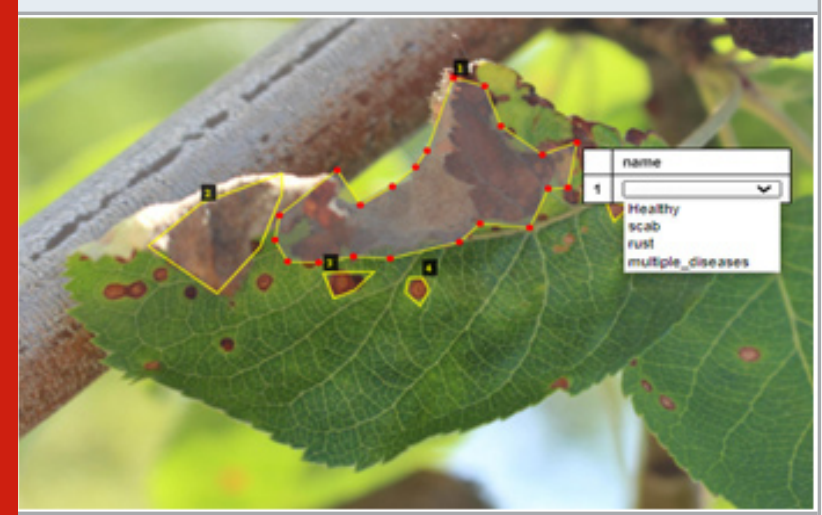

Region Proposal network: Region proposal is anarea where objects can possibly be found. It uses CNN to find regions of interest using binary classifiers. CNN layers of Regressor plot bounding boxaround possible objects and later by finding Intersection over Union we can decide which boxes possibly contain regions of interest. Region of Interest (ROI) can be calculated by dividing area of intersection by area of union.Once regions of interest get finalized, the next step is to have ROI Pooling. This 
step gets input from CNN as a feature map and Region of interests from regressor. ROI pooling is used to extract fixed size windows from feature maps that is helpful to extract labels as a final output. It will produce fixed size feature map from different size regions using max pooling and the size of max pool window will be of $7 * 7 * 512$.

\section{Experimental Setup}

Dataset: The Plant Pathology 2020 dataset is publicly available sponsored by The Cornell Initiative for Digital Agriculture and FGVC7.It has 1821 leaf images. It contains 4 classes: healthy (516 images), multiple_ diseases (91 images), rust (622 images), scab (592 images). Figure 2 shows sample images from Plant Pathology 2020 dataset.

Image Annotation: We have used VGG image Annotator to annotate each image manually. Using this tool, we obtained annotation in .json file for train and test dataset. We have kept this file in the train and test folder respectively.

Train the ResNet50 CNN for feature maps: We have trained ResNet50 CNN algorithms on The Plant Pathology 2020 dataset. We have considered feature maps of the images. Images have been resized to $224 \times 224 \times 3$ and then divided into two parts: train and test. Train data contains $80 \%$ of overall images and the rest all are in test data. To fit the data properly we normalized the image pixel values.To reduce overfitting and to improve the overall performance of the model image augmentationhas been used which will help to generalize the model. During the training phase we have used Adam optimizer with 0.001 learning rate, categorical cross entropy loss function and batch size as 10. After training we have saved weights of the model in .h5 format so that it can be used to train Mask-RCNN.

Figure 4: Confusion matrix of our model

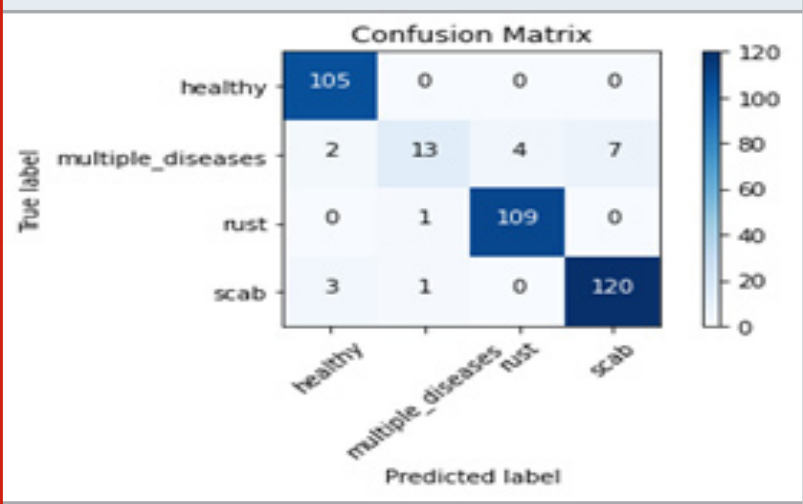

Training of Mask-RCNN: During the training phase of Mask RCNN proposed methodology uses ResNet50 as a backbone as it takes less time than ResNet101 or ResNext101 due to less numbers of layers. Detection minimum confidence set to 0.90 . Remaining all other parameters are kept as default to train the model. The algorithm is trained on NVIDIA GetForce RTX 2060 GPU with 16 GB RAM.

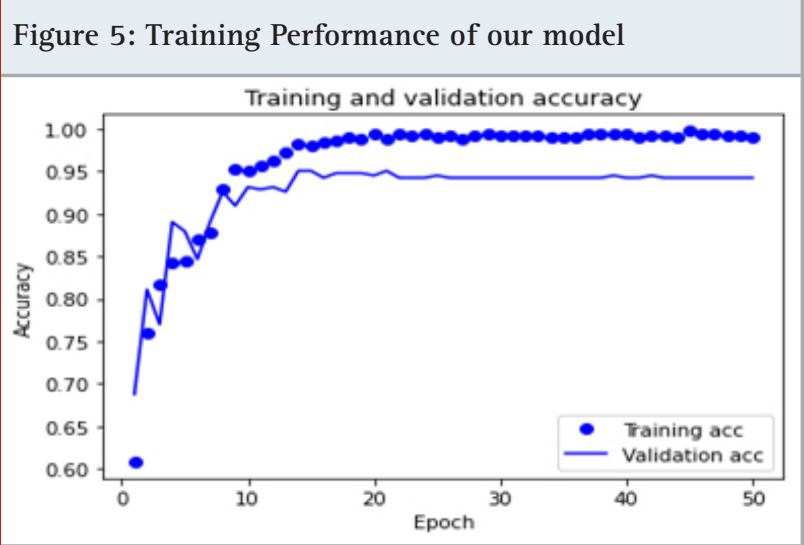

Figure 6: Learning curve of our model

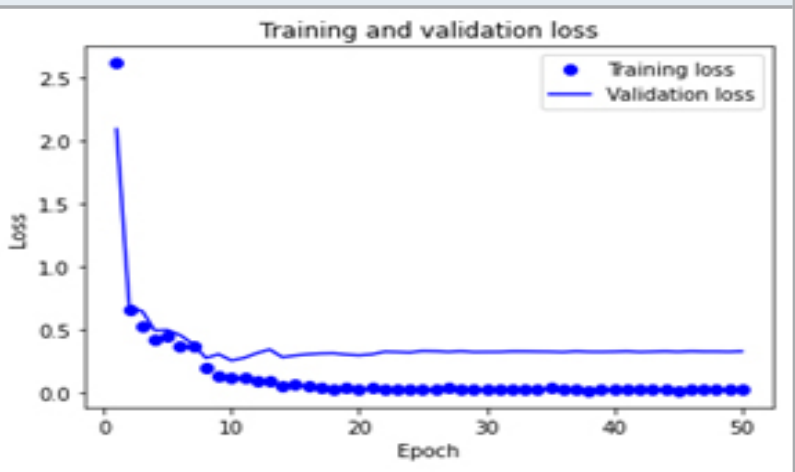

Figure 7: Predicted classes of disease with masks.

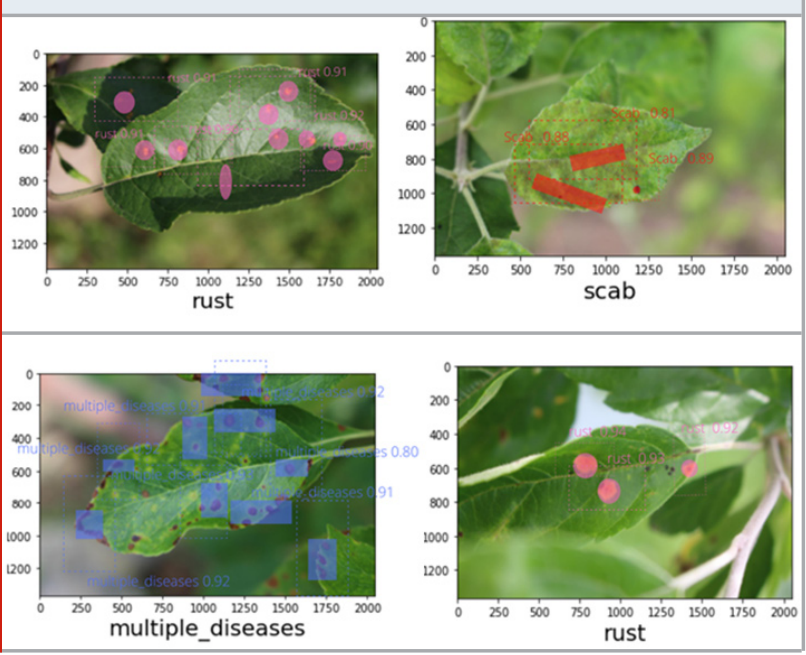

Performance measure report of ResNet50: 1We evaluated the performance of our model using a confusion matrix as shown in figure 3 and calculated accuracy, precision, recall and f1 score. ResNet50 with Plant pathology lab 2020 dataset gave 90\% accurate prediction with 0.95 precision, 0.94 recall and 0.96 as f1 score.

\section{Confusion Matrix}

Training curve and Loss curve 
Results of Mask-RCNN: As the results shown in fig algorithm performs well and can predict the disease accurately. It annotates the object(disease patches) correctly with confidence.

\section{CONCLUSION}

In this paper, we have implemented ResNet50 to obtain the weights that can be used as inputs to the proposed methodology. To validate the model, we have used a performance measures such as confusion matrix, accuracy, precision, recall and F1 score.The proposed model achieved 90\% confidence to detect each disease class. It is able to mask the affected region of the leaf with 90\% accurate labeling and can draw bounding boxes around the mask. The results indicates that our proposed model performs well with high accuracy and is able to detect apple leaf disease in an early stage in an efficient way.

\section{REFERENCES}

B. Liu, Y. Zhang, D. J. He, and Y. Li, "Identification of apple leaf diseases based on deep convolutional neural networks," Symmetry (Basel)., vol. 10, no. 1, 2018, doi: 10.3390/sym 10010011.

Data augmentation :Tensorflow core. (n.d.)". Retrieved March 12, 2021, from https://www.tensorflow.org/ tutorials/images/data_augmentation

D. P. Kingma and J. L. Ba, "Adam: A method for stochastic optimization," 3rd Int. Conf. Learn. Represent. ICLR 2015 - Conf. Track Proc., pp. 1-15, 2015.

G. Hu, X. Yang, Y. Zhang, and M. Wan, "Identification of tea leaf diseases by using an improved deep convolutional neural network," Sustain. Comput. Informatics Syst., vol. 24, p. 100353, 2019, doi: 10.1016/j.suscom.2019.100353.

K. K. Singh, "An Artificial Intelligence and Cloud Based Collaborative Platform for Plant Disease Identification, Tracking and Forecasting for Farmers," Proc. - 7th IEEE Int. Conf. Cloud Comput. Emerg. Mark. CCEM 2018, pp.
49-56, 2019, doi: 10.1109/CCEM.2018.00016.

VGG Image Annotator (VIA)." Visual Geometry Group

- University of Oxford, www.robots.ox.ac.uk/ vgg/ software/via/.

M. Bin Tahir et al., "Recognition of Apple Leaf Diseases using Deep Learning and Variances-Controlled Features Reduction," Microprocess. Microsyst., p. 104027, 2021, doi: 10.1016/j.micpro.2021.104027.

M. R-cnn, P. Doll, and R. Girshick, "Mask R-CNN."

Q. Zhang, M. Zhang, T. Chen, Z. Sun, Y. Ma, and B. $\mathrm{Yu}$, "Recent advances in convolutional neural network acceleration," Neurocomputing, vol. 323, pp. 37-51, 2019, doi: 10.1016/j.neucom.2018.09.038.

R. Girshick, J. Donahue, T. Darrell, and J. Malik, "Rich feature hierarchies for accurate object detection and semantic segmentation," Proc. IEEE Comput. Soc. Conf. Comput. Vis. Pattern Recognit., pp. 580-587, 2014, doi: 10.1109/CVPR.2014.81.

Subetha.T., R. Khilar, and M. Subaja Christo, “A comparative analysis on plant pathology classification using deep learning architecture - Resnet and VGG19," Mater. Today Proc., no. xxxx, 2021, doi: 10.1016/j. matpr.2020.11.993.

V. Singh and A. K. Misra, "Detection of plant leaf diseases using image segmentation and soft computing techniques," Inf. Process. Agric., vol. 4, no. 1, pp. 41-49, 2017, doi: 10.1016/j.inpa.2016.10.005.

Y. Guo et al., "Plant Disease Identification Based on Deep Learning Algorithm in Smart Farming," Discret. Dyn. Nat. Soc., vol. 2020, 2020, doi: 10.1155/2020/2479172.

Y. Li, J. Nie, and X. Chao, "Do we really need deep CNN for plant diseases identification?," Comput. Electron. Agric., vol. 178, no. September, p. 105803, 2020, doi: 10.1016/j.compag.2020.105803.

Y. Zhong and M. Zhao, "Research on deep learning in apple leaf disease recognition," Comput. Electron. Agric., vol. 168, no. October 2019, p. 105146, 2020, doi: 10.1016/j.compag.2019.105146. 